between sampling as widely as possible, with a consequent reduction in response rates, or sampling more narrowly and missing important pockets of data. With regard to the validity of the therapists' reports, as explained in our article, we did, in fact, tackle this issue by comparing respondents who had referred to notes with those who had not. No differences were found on any of the variables in the analyses.

In responding to our research, Drs Brandon \& Boakes refer to their own experience of therapists and individuals who clain to have recovered memories. However, they do not provide any numbers or information about the source of their data. As they will be the first to acknowledge, the people who form the basis of their experiences are unlikely to be a representative sample. In contrast, our survey involved what is, to date, the largest number of observations of clients reporting recovered memories collected systematically in a sample of therapists of all theoretical persuasions.

Andrews, D, Morton, J., Belcerian, D. A., et el (ISAS) The recovery of memories in clinical practice: experiences and beliefs of British Psychological Society Practitioners. Psychologist: Bulletin of the British Psychological Society, 5, 209-214.

B. Andrews Department of Psychology, Royal Holloway, University of London, Egham, Surrey TW20 OEX

\section{Ecstasy use and neuropathology}

Sir: Obrocki et al (1999) claim to reveal long-term effects of methylenedioxymethamphetamine (MDMA) on the central nervous system in a study of seven subjects, but in fact repeat the errors of previous published studies of neurotoxicity in humans (McCann et al, 1998). McCann et al provided convincing evidence of an association between decreased serotonin (5-HT) transporter binding sites and MDMA use, and Obrocki et al demonstrate an association between MDMA use and altered glucose metabolic uptake in Brodmann's area 11 in particular. However, association does not imply causation, and the findings could be explained in terms of neuropathology caused by MDMA use, MDMA use caused by neuropathology, or both arising from a common denominator. Both Obrocki et al and McCann et al focus exclusively on the first hypothesis to the detriment of their otherwise convincing results. As has already been noted (Morgan, 1999), it is equally plausible that MDMA use results from pre-existing 5-HT function abnormalities; in other words, affective disturbance may lead individuals to 'self-medicate' with MDMA. Since absence of psychiatric disease constituted an exclusion criterion for Obrocki's control subjects, $P$ values would be expected to be lowered further if Morgan's (1999) hypothesis were true. Future studies should consider Axis I disorder prior to MDMA use, subclinical mood disorder, and previous or current Axis II diagnoses with particular reference to impulsive behaviour in which hippocampal pathology may be involved. Since MDMA use in the community is so prevalent, prospective studies would be valuable and possible, although ethically difficult.

McCann, U D, Szabo, Z., Scheffe, U., et of (1996) Positron emission tomographic evidence of toxic effect of MDMA ("Ecstasy") on brain serotonin neurons in human beings. Loncet, 352, 1433-1437.

Morgan, J. F. (1999) Positron emission tomographic evidence of toxic effects of MDMA ('ecstasy') on brain serotonergic neurones in human beings: users may selfmedicate for axis I and II disorders. Loncet, 353. $1268-1269$.

Obrocld, J., Buchert, R., Vititerlein, O., et al (1999) Ecstasy - long-term effects on the human central nervous system revealed by positron emission tomography. British journal of Psychiotry, 175, 186-188.

J. F. Morgan Department of General Psychiatry, St George's Hospital Medical School, London SWI7 ORE

Authors' reply: We agree that there is a theoretical possibility that the alterations in glucose metabolic rates detected by positron emission tomography within the ecstasy user group might be caused by preexisting aberrant neuronal activity. Yet, to our knowledge, conclusive neuropathological findings that are characteristic for secondary drug misuse do not exist. Putative psychopathological phenomena mediating the secondary use of ecstasy are most likely heterogeneous and too diffuse with regard to the 2-( $\left.{ }^{18} \mathrm{~F}\right)$-fluoro-2-deoxy-D-glucose PET method. It therefore seems more reasonable that the detection of altered glucose metabolic rates in ecstasy users is a consequence of experimentally well-documented neurotoxic serotonergic lesions caused by MDMA. In this sense, we disagree that it is equally plausible that MDMA abuse results from measurable pre-existing 5-HT function abnormalities. We have examined the relations between psychiatric disorders and PET findings mentioned by Dr Morgan in a completed study with 107 ecstasy users (Thomasius, 1998). The publication of the results is in preparation.

Thomachus, R. (1998) Ecstasy: user groups and risk levels. An empirical study based on psychiatric-. psychodynamic-, EEG-, EP-, PET and other medical diagnostics of 100 Ecstasy users. Wiener Zeitschrift für Suchtforschung, 21, 9-14

J. Obrocki, R. Thomasius Department of Psychiatry and Psychotherapy, University Hospital, Martinistr. 52, 20246 Hamburg, Germany

\section{Selective serotonin reuptake inhibitors and personality change}

Sir: In Ekselius \& von Knorring's (1999) study, I find it difficult to accept the assumed independence between depression and the majority of the self-rated Karolinska Scales of Personality (KSP) (e.g. somatic and psychic anxiety, muscular tension, monotony avoidance, indirect aggression, inhibition of aggression, irritability, guilt, detachment, social desirability). The authors considered the Montgomery-Åsberg Depression Rating Scale (MADRS) change (baseline - after 24 weeks) as an indicator of improvement in depression, and used this variable as a single predictor in a series of stepwise regression analyses with changes in single different KSP scores as outcome variables. The consistently low coefficient of determination $R^{2}$ values lead the authors to conclude that factors other than improvement in depression must explain their findings, and speculate that there is a direct effect of selective serotonin reuptake inhibitors (SSRIs) on the personality scales. These conclusions can be challenged. I believe that MADRS change is unsuitable for solving this problem, as it expresses magnitude of change (interval level). Suppose that in a sample of 40 improved subjects who experience change in KSP, 20 show large MADRS change values and small differences for many KSP items, and the other 20 show small MADRS change values but large differences for many KSP items. Then, the correlations between MADRS change and differences in KSP will be relatively small, despite the strong correlations between KSP changes and improvement considered at a categorical level. The Clinical Global Impression scale, also measured in this study, could address this question more 